\title{
Low-temperature fluorescence spectra of greening bean leaves
}

As shown by BRoDY ${ }^{1}$ the fluorescence spectrum of chlorophyll $a$ dissolved in ethanol in high concentration shows an additional peak at $725 \mathrm{~m} \mu$ as compared with the fluorescence spectrum of a diluted chlorophyll solution. This additional band, considerably pronounced at liquid-air temperature, was ascribed to a chlorophyll dimer. The red absorption band of the chlorophyll $a$ dimer shows increased absorption both on the long-wave side and on the short-wave side of the monomer band ${ }^{3}$. In the green algae Euglena gracilis and Ochromonas danica an additional fluorescence band located at $720 \mathrm{~m} \mu$ was also measured at $-\mathrm{r} 96^{\circ}$ by BRODY AND LINSCHITz ${ }^{2}$. BRODY AND BRODY ${ }^{3}$ hence assume that in the living cell a fraction of the chlorophyll molecules occurs in a dimeric state as well, while the absorption on the long-wave side of the red chlorophyll $a$ band is ascribed to the dimer.

As dimerisation in vitro may be seen as a result of high pigment concentration, an investigation was made as to whether the occurrence of the $720-\mathrm{m} \mu$ fluorescence band is related to chlorophyll concentration in greening bean leaves. Fluorescence spectra were measured of etiolated bean leaves during greening. Immediately before the determination of the fluorescence spectrum the photosynthetic capacity of the same leaves was also measured. Fluorescence was excited by the $366-\mathrm{m} \mu \mathrm{Hg}$ line. Spectra were recorded using a Bausch and Lomb grating monochromator and a Dumont 69I I photomultiplier cooled by liquid air. The spectra are corrected for multiplier sensitivity and monochromator transmission. Photosynthesis of a single leaf was measured as oxygen production with a mass spectrometer. The leaves were placed in a Dewar vessel provided with a window and sample compartment. Spectra were recorded before and after cooling with liquid nitrogen.

Immediately after transformation of protochlorophyll into chlorophyll $a$ the fluorescence spectrum does not change in shape as a result of cooling. The fluorescence

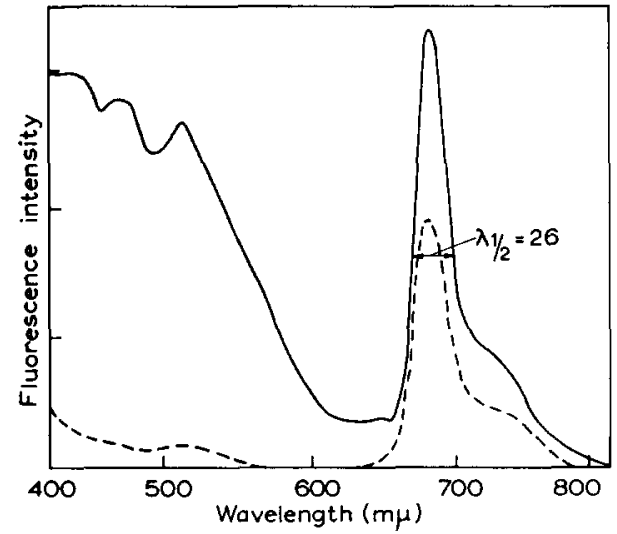

Fig. I. Fluorescence spectrum of an etiolated bean leaf resulting from excitation with $366-\mathrm{m} \mu$ $\mathrm{Hg}$ light. The leaf was allowed ${ }_{5} \mathrm{~min}$ to remain in the light before the fluorescence spectrum was taken (dashed line). Immediately afterwards, the leaf was cooled with liquid nitrogen and the fluorescence spectrum was redetermined (solid line). Slit width of monochromator, $4 \mathrm{~m} \mu$.

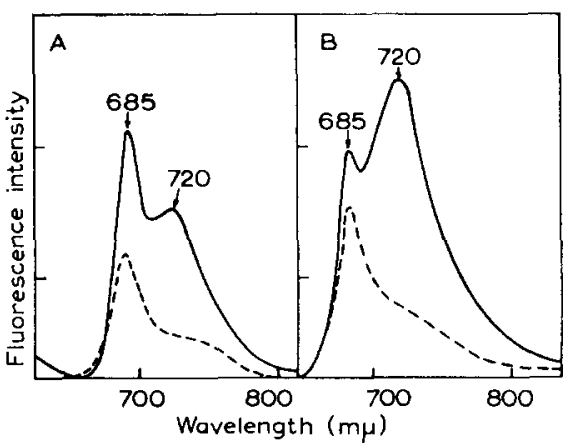

Fig. 2. Fluorescence spectra of etiolated bean leaves at room temperature $\left(23^{\circ}\right.$, dashed line) and liquid-nitrogen temperature (solid line) after 2.5 -h (A) and $3.5^{-h}$ (B) storage in light (400 $\mathrm{ft}$ candles incandescent light). With the leaves under (A) no photosynthesis could be measured, with the leaves under (B) photosynthesis could be observed. 
intensity, however, is doubled at each wavelength. If a leaf is allowed to remain at room temperature for approx. I5 min the fluorescence maximum is shifted from 694 to $683 \mathrm{~m} \mu$ but once again cooling does not result in a change in shape (Fig. I). It thus appears that neither the $684-\mathrm{m} \mu$ and the $673-\mathrm{m} \mu$ forms of chlorophyll occurring consecutively upon protochlorophyll transformation ${ }^{4}$ are responsible for the $720-\mathrm{m} \mu$ fluorescence peak after cooling. In healthy young leaves, and without preillumination, formation of new protochlorophyll does not occur until $2.5 \mathrm{~h}$ after the start of illumination, while also no oxygen production could be measured within this time ( $c f$. also SMiтH $^{5}$ ). In Fig. 2, fluorescence spectra after 2.5 and $3.5 \mathrm{~h}$ of illumination are given. According to the absorption measurements (opal-glass method) the chlorophyll $a$ content of the leaves was approximately doubled between 2.5 and $3.5 \mathrm{~h}$. After $2.5 \mathrm{~h}$ of illumination only a slight change in shape of the fuorescence spectrum was noticed

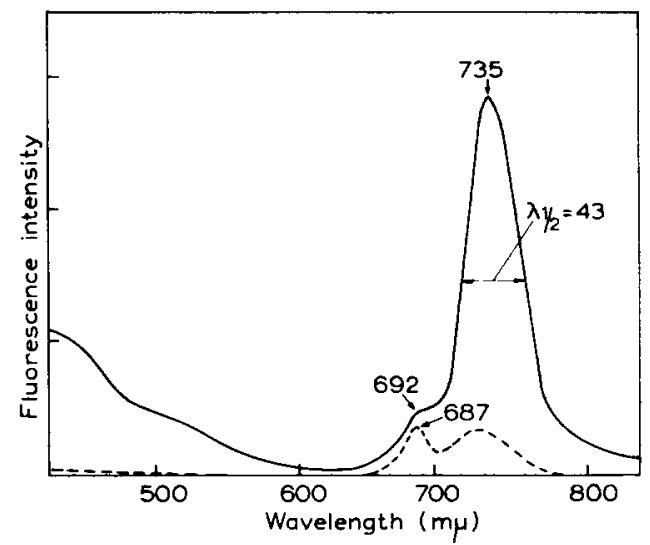

Fig. 3. Fluorescence spectrum of a bean leaf after $40 \mathrm{~h}$ of greening. Half-width value of the $735-\mathrm{m} \mu$ band is approx. I. 7 times half-width value of the $680-\mathrm{m} \mu$ band in Fig. 1 . Blue fluorescence spectrum shows less marked bands than spectrum of etiolated leaves. Room-temperature spectrum is shown by dashed line, low-temperature spectrum by solid line.

at low temperatures. The " $3 \cdot 5-\mathrm{h}$ " leaf showed a marked $720-\mathrm{m} \mu$ fluorescence band. Only after $3.5 \mathrm{~h}$ a weak photosynthesis (at light saturation about $6 \%$ of that of a comparable green leaf) could be measured. During further greening more chlorophyll develops, accompanied by an increase of both oxygen production and height of the 720-m $\mu$ fluorescence band.

After $40 \mathrm{~h}$ of greening the amount of chlorophyll in the leaf was about $5^{\circ}$ times that after the first illumination. With respect to the room-temperature spectrum of a fully green leaf we have indications, derived from induction phenomena, that the high value of fluorescence around $720 \mathrm{~m} \mu$ in such a leaf is not merely a result of light scattering and reabsorption in the major peak by high pigment concentration (VIRGIN ${ }^{6,7}$ found these mechanisms to be of great importance for the shape of the fluorescence spectrum of a green leaf) but has to be ascribed partly to emission by the chlorophyll $a$ complex responsible for the additional low-temperature band. At liquid-nitrogen temperatures the intensity of this additional band (shifted in the fully green leaf to $735 \mathrm{~m} \mu$ ) is about II times the intensity at this wavelength at room temperature, while, at $690-\mathrm{m} \mu$ fluorescence, is increased approx. $50 \%$.

Figs. I and 3 also show fluorescence in the blue region of the spectrum. This 
blue fluorescence also is increased markedly as a result of cooling. We tentatively ascribe the 520-m $\mu$ fluorescence band to FMN emission (an afterglow of the characteristic color of FMN luminescence can be seen after cooling) while part of the fluorescence around $45^{\circ} \mathrm{m} \mu$ probably is caused by DPNH or derivates 8 .

Development of the $720-\mathrm{m} \mu$ fluorescence seems to be related more to development of photosynthetic capacity than to chlorophyll concentration.

In two experiments, the leaves had received a 3 -min preillumination with $645-\mathrm{m} \mu$ light of $\mathrm{I} 50 \mathrm{ergs} \cdot \mathrm{cm}^{-2} \cdot \mathrm{sec}^{-1}$. When $\mathrm{r} 8 \mathrm{~h}$ later continuous illumination was started, greening proceeded nearly instantaneously, while oxygen evolution could be measured only after $2 \mathrm{~h}$. The leaves contained much more chlorophyll than those of Fig. $2 \mathrm{~b}$, but no $720-\mathrm{m} \mu$ fluorescence band could be observed. This may indicate that, if the $720-\mathrm{m} \mu$ band is to be ascribed to dimer fluorescence, the onset of photosynthetic capacity is correlated with dimer formation. This dimer formation may be caused by an internal rearrangement of chlorophyll molecules, occurring at the development of lamellar chloroplast structure from the grid-like structure in etiolated chloroplasts.

This study thus provides experimental support for the hypothesis made by Brody ${ }^{3}$ that the chlorophyll type responsible for $7^{20}-\mathrm{m} \mu$ fluorescence is essential in photosynthesis. It need not necessarily be that this type participates directly in the photochemical processes. This chlorophyll type might act for example as an agent in separating products resulting from photochemical reactions along the lamellar systems or in protecting the pigment apparatus against photooxidation.

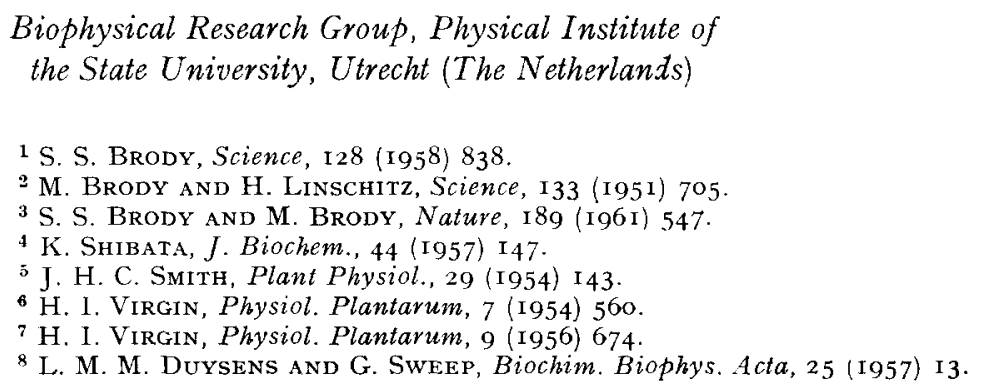

J. C. GoedheER

Received July i Ith, I96I

Biochim. Biophys, Acta, 53 (1961) 420-422

\section{Composition of brain gangliosides}

Brain gangliosides were for a long time considered to contain a uniform carbohydrate moiety ${ }^{1}$. However, by applying chromatography on cellulose one of us was able to separate human-brain gangliosides into two main fractions ${ }^{2,3}$. From the faster-moving fraction the gangliosides were crystallized for which following sequences of constituents were proposed:

ceramide-hexose-hexose- $N$-acetylgalactosamine- $N$-acetylneuraminic acid 
The occurrence of hexosamine-free gangliosides was also suggested ${ }^{3,4}$. This assumption has recently been verified by KLENK AND GIELEN ${ }^{5}$, who succeeded in isolating hexosamine-free gangliosides.

From cow-brain gangliosides $\mathrm{KUHN}^{6}$ and associates have isolated fast-and slowmoving gangliosides by chromatography on cellulose. The fast-moving gangliosides were shown to contain a tetrasaccharide for which a tentative structure was proposed. The slow-moving gangliosides could be separated into three distinct components, which were said to have a more complex structure. We have studied the slow-moving fractions of human- and calf-brain gangliosides during the last few years and have been able to show that they contain two moles of sialic acid per mole ceramide.

A crude ganglioside fraction was isolated as described earlier ${ }^{2}$. It was purified by chromatography on activated silicic acid (Baker A.R.) passing 300 mesh. The following elution scheme has been used for the isolation of total gangliosides: chloroform-methanol ( $9: \mathrm{I}, \mathrm{v} / \mathrm{V}), 5$ column volumes; $(4: \mathrm{I}), 5$ vol.; $(2: \mathrm{I}), 5$ vol. and $(\mathrm{I}: 2)$, ro vol. The eluate was collected on an automatic fraction collector, and the separation of the lipids examined by paper partition chromatography ${ }^{7}$ and qualitative tests for sialic acid. All fractions containing sialic acid-positive material were pooled and evaporated to a small volume. The gangliosides were then precipitated with large volumes of acetone. The separation of gangliosides in fast- and slow-moving ones was achieved by repeated chromatography on silicic acid with a modified elution scheme: chloroform-methanol ( $9: I$ ), 5 vol.; (4:I), 5 vol.; (3:2), Io vol. and (I:2), Io vol. The fast-moving gangliosides were eluted with chloroform-methanol $(3: 2)$ and, with some overlapping, the slow-moving gangliosides with chloroform-methanol $(\mathrm{I}: 2)$. There were about equal amounts of the two fractions in adult human and calf brains. The carbohydrate composition of human- and calf-brain gangliosides is given in Table I.

The fatty acids of the human-brain gangliosides have been investigated by Dr. S. Stenhagen with gas-phase chromatography. She found about $85 \%$ stearic

\section{TABLE I}

CARBOHYDRATE COMPOSITION OF GANGLIOSIDES

\begin{tabular}{|c|c|c|c|}
\hline Material & $\begin{array}{c}\text { Galactosamine } \\
(\%)\end{array}$ & $\begin{array}{c}\text { Hexose } \\
(\%)\end{array}$ & $\begin{array}{c}\text { N-acetyl- } \\
\text { neuraminic acid } \\
(\%)\end{array}$ \\
\hline \multicolumn{4}{|l|}{ Human gangliosides } \\
\hline Total & $7 \cdot 9$ & $23 \cdot 7$ & 24.0 \\
\hline Fast moving & 9.7 & 27.4 & Ig. 8 \\
\hline Slow moving & 9.9 & 24.7 & 3 I. 2 \\
\hline Tay-Sachs' brain & I I 5 & 21.6 & $2 \mathbf{I} .2$ \\
\hline \multicolumn{4}{|l|}{ Calf gangliosides } \\
\hline Total ${ }^{\star}$ & $9 \cdot 3$ & $24 \cdot 3$ & 23.7 \\
\hline Fast moving & 6.8 & 28.2 & I 9.4 \\
\hline Slow moving & 8.3 & 20.2 & 32.0 \\
\hline
\end{tabular}

"This preparation contained relatively large amounts of a neutral aminoglycolipid, which contained $16 \%$ of galactosamine. 
acid in fast- and slow-moving gangliosides and in the gangliosides of the Tay-Sachs' brain. The pattern of the other fatty acids was also the same in the three different ganglioside preparations.

The molar ratio sialic acid to sphingosine was I in the fast-moving gangliosides and nearly 2 in the slow-moving ones. They have provisionally been termed monoand disialogangliosides, respectively.

The disialogangliosides were converted to monosialogangliosides by mild acid hydrolysis $\left(0.05 \mathrm{~N} \mathrm{HCl}\right.$ at $80^{\circ}$ for $\left.30 \mathrm{~min}\right)$ or by enzymic hydrolysis with neuraminidase. In the acid hydrolysis small amounts of sialic acid-free glycolipids were formed, but this did not occur in the enzymic hydrolysis.

At present the following composition of the carbohydrate moieties in humanbrain gangliosides is assumed:

$\begin{array}{lll}\text { Monosialogangliosides } & \text { Hex-Nacgal } & \text { Hex-Hex } \\ & \text { Hex-Nana } & \text { Hex-Nana } \\ \text { Disialogangliosides } & \text { Hex-Nacgal } & \text { Hex-Hex } \\ & \text { Hex-Nana } & \text { Hex-Nana } \\ & \text { Nana } & \text { Nana }\end{array}$

Hex $=$ Hexose $;$ Nacgal $=N$-acetylgalactosamine $;$ Nana $=N$-acetylneuraminic acid .

(The position of attachment of the carbohydrate and ceramide moieties is from $\mathrm{C}-\mathrm{I}$ of hexose to C-I of sphingosine.)

Besides these two different types of monosialogangliosides a third type has been indicated in the chromatograms, but the composition of its carbohydrate moiety is quite unknown.

The gangliosides from a case with Tay-Sachs' disease were up to more than $90 \%$ of an abnormal type. They were monosialogangliosides but they had a much higher $R_{\boldsymbol{F}}$-value in alkaline, neutral and acidic solvents in chromatography on paper or on silica-gel plates than the normal monosialogangliosides. The ganglioside fraction also contained large amounts of an aminoglycolipid lacking sialic acid ( $N$-acylsphingosinedihexosido- $N$-acetylhexosamine), which had a much higher $R_{F}$ value than the corresponding neutral aminoglycolipid from normal brains.

\footnotetext{
Department of Medical Biochemistry, University of Gothenburg, Gothenburg (Sweden)

LARS SVEnNERHolm AINO RAAL
}

1 E. Klenk and K. Lauenstein, Z. physiol. Chem. Hoppe-Seyler's, 295 (1953) I64.

2 L. SVENNERHOLM, Nature, I 77 (1956) 524.

${ }^{3}$ L. Svennerholm, in J. N. Cumings, Cerebral Lipidoses, Blackwell, Oxford, 1957, p. 139.

4 L. Svennerholm, Acta Soc. Med. Upsaliensis, 62 (1957) I.

5 E. Klenk and W. Gielen, Z. physiol. Chem. Hoppe-Seyler's, 323 (1961) I 26.

6 R. Kunn, Angew. Chem., 72 (1960) 805.

7 U. Beiss and O. Armbruster, Z. Naturforsch., I3b (1958) 79.

Received July 26 th, I 96 I 